\title{
QCD thermodynamics with Wilson fermions
}

\section{Szabolcs Borsányi ${ }^{a}$, Zoltán Fodor ${ }^{a b c}$, Christian Hoelbling ${ }^{a}$, Sándor D. Katz ${ }^{c}$, Stefan Krieg $^{a b}$, Dániel Nógrádi ${ }^{*}$, Bálint C. Tóth ${ }^{a}$, Kálmán K. Szabó ${ }^{a}$, Norbert Trombitás ${ }^{c}$}

${ }^{a}$ Department of Physics, University of Wuppertal, Gaußstrasse 20, D-42119, Germany

${ }^{b}$ Jülich Supercomputing Centre, Forschungszentrum Jülich, D-52425 Jülich, Germany

${ }^{c}$ Institute of Physics, Eötvös University, Pázmány Péter sétány 1/a, Budapest 1117, Hungary

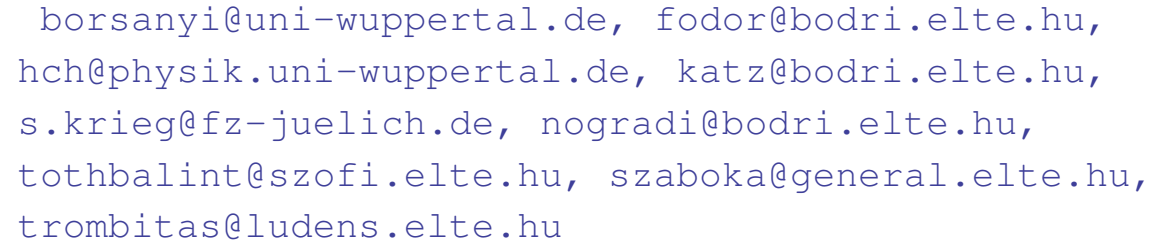

QCD is investigated at finite temperature using Wilson fermions in the fixed scale approach. A $2+1$ flavor stout and clover improved action is used at four lattice spacings allowing for control over discretization errors. The light quark masses in this first study are fixed to heavier than physical values. The renormalized chiral condensate, quark number susceptibility and the Polyakov loop is measured and the results are compared with the staggered formulation in the fixed $N_{t}$ approach. The Wilson results at the finest lattice spacing agree with the staggered results at the highest $N_{t}$.

The XXIX International Symposium on Lattice Field Theory - Lattice 2011

July 10-16, 2011

Squaw Valley, Lake Tahoe, California

\footnotetext{
*Speaker.
} 


\section{Introduction}

Extensive experimental work is currently being done with heavy ion collisions to study the QCD transition, most recently at the Relativistic Heavy Ion Collider, RHIC and at the Large Hadron Collider, LHC. Both for the cosmological transition and for RHIC/LHC, the net baryon densities are quite small, thus the baryonic chemical potentials $(\mu)$ are much less than the typical hadron masses, $\mu$ is below $50 \mathrm{MeV}$ at RHIC, even smaller at LHC and negligible in the early universe. Thus in this study we stay at $\mu=0$; for a review on thermodynamics see [1].

When one analyzes the absolute scale or any other question related to the $T>0$ QCD transition for the physically relevant case two ingredients are quite important.

First of all, one should use physical quark masses. The nature of the transition strongly depends on the quark mass. Lattice studies and effective models showed that in the three flavor theory for small or large quark masses the transition is a first order phase transition, whereas for intermediate quark masses it is an analytic crossover.

Secondly, the nature and other characteristics of the $T>0$ QCD transition is known to suffer from discretization errors $[2,3]$. Let us mention one example which underlines the importance of removing these discretization effects by performing a controlled continuum extrapolation. The three flavor theory with a large, $a \approx 0.3 \mathrm{fm}$ lattice spacing and standard staggered action predicts a critical pseudoscalar mass of about $300 \mathrm{MeV}$ [4]. This point separates the first order and cross-over regions. If we took another discretization, with another discretization error, the critical pseudoscalar mass turns out to be much smaller, well below the physical pion mass of $135 \mathrm{MeV}$. The only way to determine the physical features of the transition is to carry out a careful continuum limit analysis. It can be safely done only in the so-called scaling regime. To carry out a controlled continuum extrapolation at least three lattice spacings in the scaling regime are needed, because two points will always lie on a 2-parameter curve describing the corrections to the continuum results.

Numerically it is very demanding to fulfill both conditions. There are only a few cases for which this has been achieved. However it is important to note that fulfilling the second condition (at least 3 lattice spacings) without fulfilling the first one (physical quark masses) still leads to universal results. In other words continuum extrapolated results with non-physical quark masses are universal. These results are not the same as they are for physical quark masses, but they are well defined and unique. Contrary to this universality, fulfilling the first condition (physical quark mass) but not the second one (at least 3 lattice spacings) leads to non-universal, non-physical results. These results still have unknown discretization errors.

For this reason in this first study of QCD thermodynamics with Wilson fermions we have chosen to work at heavier than physical quark masses but simulate at four lattice spacings in order to approach the continuum limit.

In this paper we determine the temperature dependence of the chiral condensate, strange quark number susceptibility and Polyakov-loop in 2+1 flavor QCD. We use Wilson fermions with six stout smearing and tree-level clover improvement in the quark sector and tree-level improved fields in the gauge sector; for the details of the action see [5].

The structure of the paper is as follows. After this brief introduction the main features of the action and run parameters are listed in Section 2. Our choice of renormalization procedures for the 


\begin{tabular}{|c|c|c|c|c|}
\hline$\beta$ & $a m_{\text {ud }}$ & $a m_{s}$ & $N_{s}$ & $N_{t}$ \\
\hline \hline 3.30 & -0.0985 & -0.0710 & 32 & $4-12,32$ \\
\hline 3.57 & -0.0260 & -0.0115 & 32 & $4-16,64$ \\
\hline 3.70 & -0.0111 & 0.0 & 48 & $8-28,48$ \\
\hline 3.85 & -0.00336 & 0.0050 & 64 & $12-28,64$ \\
\hline
\end{tabular}

Table 1: Simulation parameters. The $N_{t}$ values used for the finite temperature runs and the values used for the zero temperature runs are separated by a comma. For finite temperature only even $N_{t}$ values were used.

\begin{tabular}{|c|c|c|c|c|}
\hline$\beta$ & $m_{\pi} / m_{\Omega}$ & $m_{K} / m_{\Omega}$ & $\left(2 m_{K}^{2}-m_{\pi}^{2}\right) / m_{\pi}^{2} \approx m_{s} / m_{u d}$ & $a[\mathrm{fm}]$ \\
\hline \hline 3.30 & $0.336(5)$ & $0.378(6)$ & $1.53(4)$ & $0.137(2)$ \\
\hline 3.57 & $0.314(3)$ & $0.351(4)$ & $1.50(2)$ & $0.095(2)$ \\
\hline 3.70 & $0.317(2)$ & $0.356(3)$ & $1.53(3)$ & $0.0727(5)$ \\
\hline 3.85 & $0.322(6)$ & $0.362(6)$ & $1.52(6)$ & $0.0567(9)$ \\
\hline
\end{tabular}

Table 2: Mass ratios from spectroscopy. The lattice spacings are given by $m_{\Omega}=1672 \mathrm{MeV}$.

various measured quantities are summarized in Section 3. The results are given in Section 4. In Section 5 we summarize and provide a short outlook.

\section{Simulation points and techniques}

The gauge action is the tree level improved Symanzik action [6], the fermionic action is a $2+1$ flavor stout [7] and clover [8] improved Wilson action. The number of smearing steps is six and the smearing parameter is set at 0.11 ; for the first large scale simulation employing stout smearing see [11]. The clover improvement is tree level, i.e. $c_{s w}=1$. In order to speed up the molecular dynamics multiple time scales [9], Omelyan integrator [10] and even-odd preconditioning was used. For further details on the algorithm see [5] . The finite temperature simulations were performed in the fixed scale approach [12].

The simulation parameters are summarized in Table 1. The simulations were performed at four lattice spacings and dimensionless ratios of hadron masses were used to define the line of constant physics. The mass of the $\Omega$ baryon, $m_{\Omega}=1672 \mathrm{MeV}$, was used to set the scale. The ratio of the pion and omega mass was kept at heavier than physical value, while the mostly light quark mass independent combination $\left(2 m_{K}^{2}-m_{\pi}^{2}\right) / m_{\Omega}^{2}$ was kept close to its physical value. More precisely the combination which was tuned the most precisely is $\left(2 m_{K}^{2}-m_{\pi}^{2}\right) / m_{\pi}^{2}$ which can also be used to define a physical ratio $m_{s} / m_{u d}$ while $m_{s}$ itself was fixed by the requirement that as $m_{u d}$ is lowered to the physical point the kaon mass becomes physical too. The large lattice volumes make sure finite volume effects are negligible, in all cases $m_{\pi} L>7.9$. Table 2 summarizes the zero temperature hadron spectroscopy results. For more details see [13, 14, 15].

\section{Renormalization}

Three quantities were measured, the chiral condensate $(\langle\bar{\psi} \psi\rangle)$, the strange quark number sus- 
ceptibility $\left(\chi_{s}\right)$ and the Polyakov loop $(P) . \chi_{s}$ does not require renormalization but the others do.

\subsection{Chiral condensate}

In this section the additive and multiplicative renormalization of the chiral condensate is summarized briefly, for more details see [16] which is based on [17].

The additive divergence in the bare condensate is $O\left(a^{-3}\right)$ which at finite temperature can be removed by subtracting the zero temperature value. The multiplicative divergence also needs to be removed by multiplying an appropriate expression of the quark masses. This step is complicated by the fact that Wilson fermions break chiral symmetry explicitly but nevertheless the axial Ward identity ensures that the following two combinations are finite and agree in the continuum limit [16],

$$
\begin{aligned}
& m_{R}\langle\bar{\psi} \psi\rangle_{R}(T)=m_{P C A C} Z_{A} \Delta_{\bar{\psi} \psi}(T)+O(a) \\
& m_{R}\langle\bar{\psi} \psi\rangle_{R}(T)=2 N_{f} m_{P C A C}^{2} Z_{A}^{2} \Delta_{P P}(T)+O(a)
\end{aligned}
$$

where $Z_{A}$ is a finite renormalization constant,

$$
\begin{aligned}
\Delta_{\bar{\psi} \psi}(T) & =\left\langle\bar{\psi}_{0} \psi_{0}\right\rangle(T)-\left\langle\bar{\psi}_{0} \psi_{0}\right\rangle(T=0) \\
\Delta_{P P}(T) & =\int d^{4} x\left\langle P_{0}(x) P_{0}(0)\right\rangle(T)-\int d^{4} x\left\langle P_{0}(x) P_{0}(0)\right\rangle(T=0)
\end{aligned}
$$

are the subtractions needed to cancel the additive divergences and $\left\langle\bar{\psi}_{0} \psi_{0}\right\rangle$ and $P_{0}(x)$ are the bare condensate and bare pseudo scalar density, respectively [16]. In this first study we did not measure $Z_{A}$ but rather used the two expressions (3.1) to eliminate $Z_{A}$ (and also $m_{P C A C}$ ) and end up with the finite expression

$$
m_{R}\langle\bar{\psi} \psi\rangle_{R}(T)=\frac{\Delta_{\bar{\psi} \psi}^{2}(T)}{2 N_{f} \Delta_{P P}(T)}+O(a)
$$

\subsection{Polyakov loop}

The divergence of the bare free energy means that the Polyakov loop also needs to be renormalized. A convenient choice of renormalization prescription is demanding a fixed value $P_{*}$ for the renormalized Polyakov loop at a fixed but arbitrary temperature $T_{*}>T_{c}$. Then the renormalized Polyakov loop $P_{R}$ is given by

$$
P_{R}(T)=\left(\frac{P_{*}}{P_{0}\left(T_{*}\right)}\right)^{\frac{T_{*}}{T}} P_{0}(T)
$$

in terms of the bare Polyakov loop $P_{0}(T)$. We choose $T_{*}=0.143 m_{\Omega}$ and $P_{*}=1.2$. Other choices would simply correspond to other renormalization schemes [20].

\section{Results}

We have measured three quantities at four lattice spacing. The renormalized chiral condensate is sensitive to the chiral transition whereas the renormalized Polyakov loop and the strange quark number susceptibility are sensitive to the remnant of the confinement-deconfinement transition. 


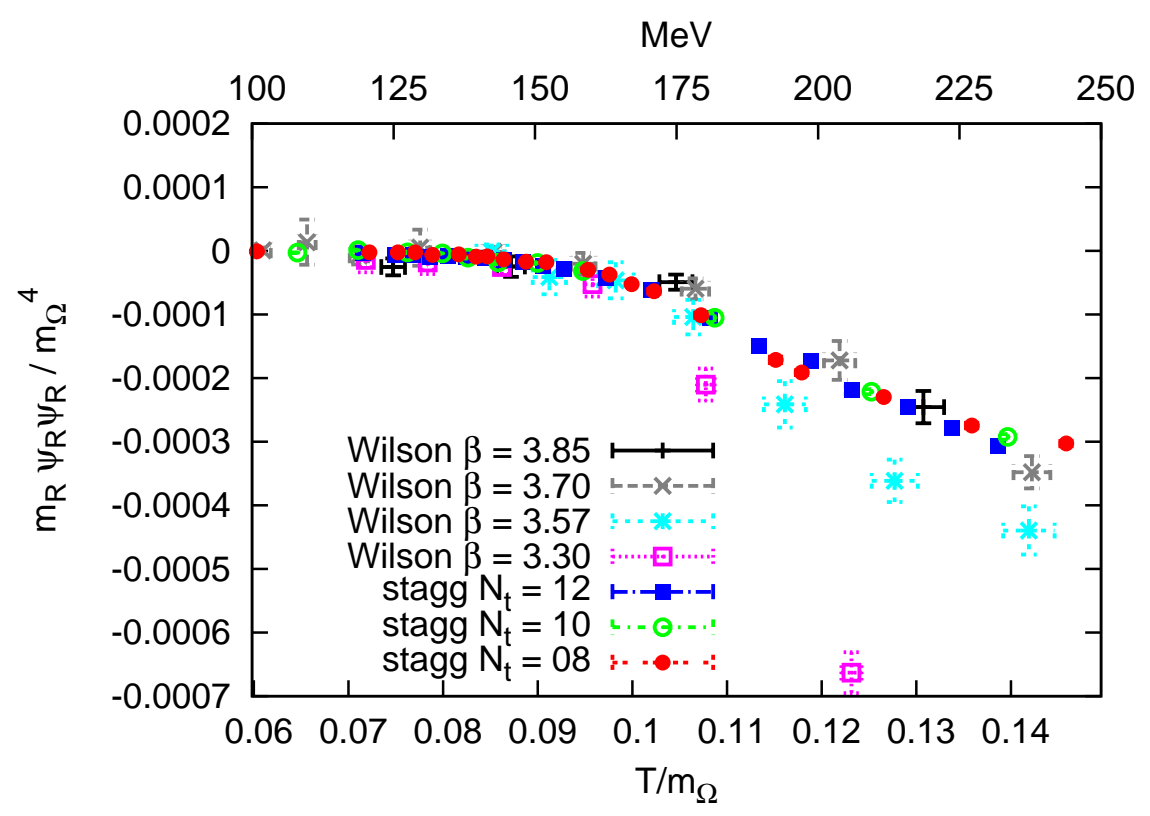

Figure 1: Renormalized chiral condensate. The four lattice spacings with Wilson fermions (in the fixed scale approach) are compared with three lattice spacings with staggered fermions in the fixed $N_{t}$ approach.

Finite continuum results should agree between different discretizations provided the same renormalization conditions are used. We have imposed the same conditions on staggered simulations hence these can be compared with the Wilson results; for a recent review on staggered thermodynamics see [18].

Figure 1 shows the renormalized chiral condensate at the four different lattice spacings together with staggered results at three fixed $N_{t}$ values, 8, 10 and 12 (the staggered results were obtained using the fixed $N_{t}$ approach, for more details see [19, 20, 21, 22]). As is apparent from the figure the two finer lattice spacing values are small enough to observe only a small change in the chiral condensate at any temperature. The rougest lattice spacing on the other hand is seen to include large discretization effects and is probably not in the scaling region. What is also clear is that results at $N_{t}<8$ are not trustworthy at any of the lattice spacings due to a too rough resolution of the imaginary time direction (temperature). At only moderately high and small temperatures, particularly around $T_{c}$, the three finest lattice spacings are probably in the scaling region and continuum extrapolation is possible.

The strange quark number susceptibility is a sum of two contributions, the connected and disconnected terms. The disconnected part is a very noisy quantity (as usual) and a large number of random vectors are needed in order to evaluate it precisely [23]. The results for the four lattice spacings are shown on Figure 2 together with the staggered results. We plot $\chi_{s} / T^{2}$ normalized by its Stefan-Boltzman limit value.

The third quantity we measured is the renormalized Polyakov loop which is sensitive to the confinement-deconfinement transition similarly to the quark number susceptibility. The results are shown on Figure 3. 


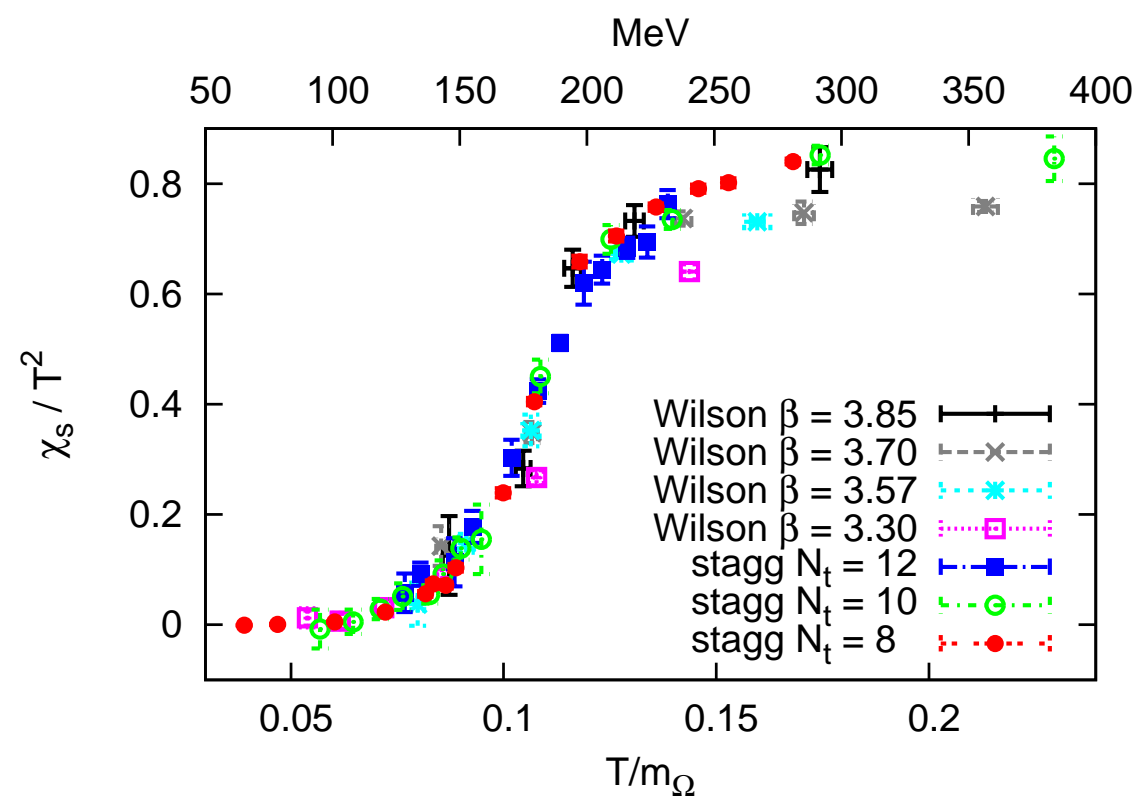

Figure 2: Strange quark number susceptibility. The four lattice spacings with Wilson fermions (in the fixed scale approach) are compared with three lattice spacings with staggered fermions in the fixed $N_{t}$ approach.

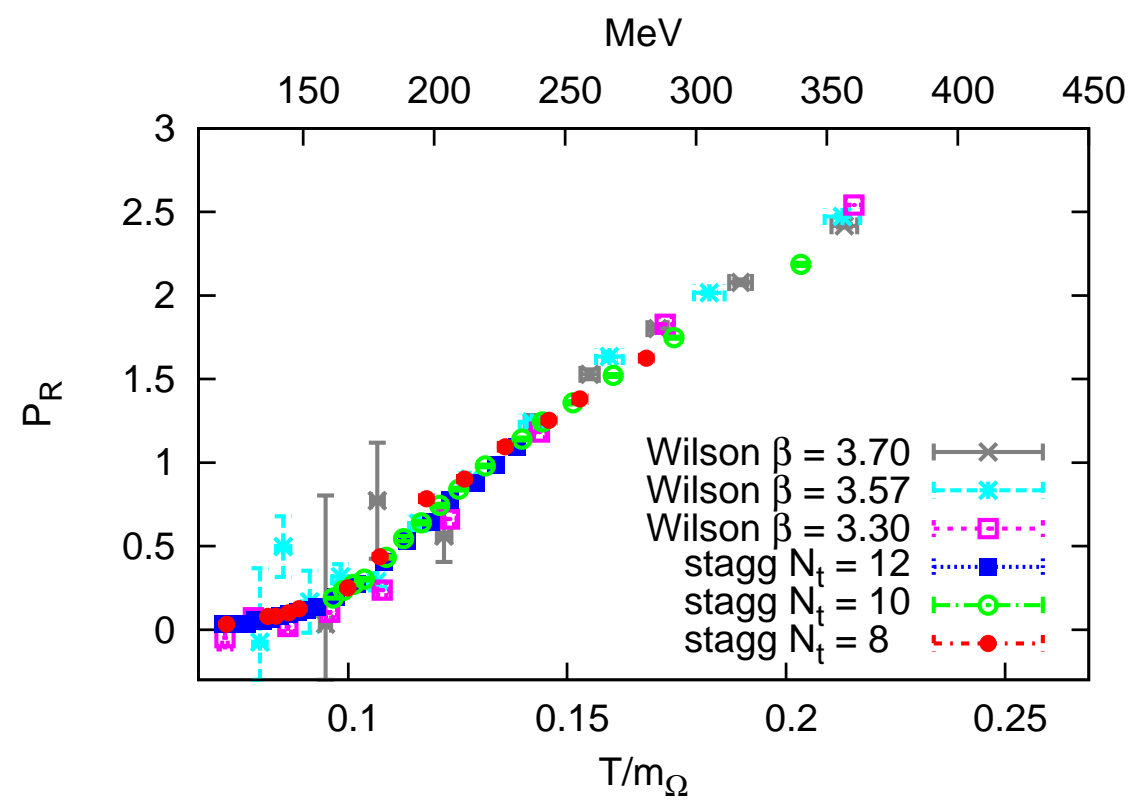

Figure 3: Renormalized Polyakov loop. The four lattice spacings with Wilson fermions (in the fixed scale approach) are compared with three lattice spacings with staggered fermions in the fixed $N_{t}$ approach. 


\section{Summary}

In this work we have started our study of $2+1$ flavor QCD thermodynamics with Wilson fermions. Special emphasis is placed on obtaining results in the continuum limit, i.e. the simulations are performed at four lattice spacings. In this first study the light quark masses were kept above the physical value but since continuum results are universal even at non-physical quark masses a comparison is possible with continuum staggered results. We found nice agreement between the finest lattice spacings of the Wilson and staggered formulations. In the future we plan to extend our results to lower pion masses and also plan to use (3.1) for $\langle\bar{\psi} \psi\rangle$ after measuring $Z_{A}$ and $m_{P C A C}$ because a priori it is not clear which formula has the better scaling property.

\section{Acknowledgment}

This work was supported by the DFG grants FO 502/1 and SFB-TR/55, and the EU Framework Programme 7 grant (FP7/2007-2013)/ERC No 208740. For our calculations we used computer time awarded by PRACE on Jugene in Juelich as well as GPU clusters in Budapest and Wuppertal.

\section{References}

[1] Z. Fodor, S. D. Katz, [arXiv:0908.3341 [hep-ph]].

[2] P. de Forcrand, S. Kim and O. Philipsen, PoS LAT2007, 178 (2007) [arXiv:0711.0262 [hep-lat]].

[3] G. Endrodi, Z. Fodor, S. D. Katz and K. K. Szabo, PoS LAT2007, 182 (2007) [arXiv:0710.0998 [hep-lat]].

[4] F. Karsch, E. Laermann and C. Schmidt, Phys. Lett. B 520, 41 (2001) [arXiv:hep-lat/0107020].

[5] S. Durr et al., Phys. Rev. D 79, 014501 (2009) [arXiv:0802.2706 [hep-lat]].

[6] K. Symanzik, Nucl. Phys. B 226 (1983) 187.

[7] C. Morningstar and M. J. Peardon, Phys. Rev. D 69 (2004) 054501 [arXiv:hep-lat/0311018].

[8] B. Sheikholeslami and R. Wohlert, Nucl. Phys. B 259 (1985) 572.

[9] J. C. Sexton and D. H. Weingarten, Nucl. Phys. B 380 (1992) 665.

[10] T. Takaishi and P. de Forcrand, Phys. Rev. E 73 (2006) 036706 [arXiv:hep-lat/0505020].

[11] Y. Aoki, Z. Fodor, S. D. Katz, K. K. Szabo, JHEP 0601, 089 (2006). [hep-lat/0510084].

[12] T. Umeda et al., Phys. Rev. D 79, 051501 (2009) [arXiv:0809.2842 [hep-lat]].

[13] S. Durr et al., Science 322, 1224 (2008) [arXiv:0906.3599 [hep-lat]].

[14] S. Durr et al, Phys. Lett. B701, 265-268 (2011). [arXiv:1011.2403 [hep-lat]].

[15] S. Durr et al., JHEP 1108, 148 (2011). [arXiv:1011.2711 [hep-lat]].

[16] L. Giusti et al., Nucl. Phys. B538 (1999) 249-277. [hep-lat/9807014].

[17] M. Bochicchio et al., Nucl. Phys. B262 (1985) 331.

[18] A. Bazavov et al., [arXiv:1111.1710 [hep-lat]].

[19] Y. Aoki, G. Endrodi, Z. Fodor, S. D. Katz and K. K. Szabo, Nature 443 (2006) 675 [arXiv:hep-lat/0611014].

[20] Y. Aoki et al., Phys. Lett. B 643, 46 (2006) [arXiv:hep-lat/0609068].

[21] Y. Aoki et al., JHEP 0906, 088 (2009) [arXiv:0903.4155 [hep-lat]].

[22] S. Borsanyi et al., JHEP 1009, 073 (2010) [arXiv:1005.3508 [hep-lat]].

[23] S. Ejiri et al. [WHOT-QCD Collaboration], Phys. Rev. D 82, 014508 (2010) [arXiv:0909.2121 [hep-lat]]. 